\title{
Vertebral level of Tuffier's line measured by ultrasonography in parturients in the lateral decubitus position
}

\author{
Se Hee Kim, Dong Yeon Kim, Jong In Han, Hee Jung Baik, Hahck Soo Park, Guie Yong Lee, and \\ Jong Hak Kim
}

Department of Anesthesiology and Pain Medicine, Ewha Womans University School of Medicine, Seoul, Korea

Background: It is known that Tuffier's line intersects the spine at the L4 spinous process or at the L4-L5 intervertebral space. Full term parturient women undergo various physical changes. Therefore, determining the vertebral level with Tuffier's line based on palpation inevitably is not very accurate. The aim of this study was to use ultrasound to verify the difference between vertebral levels for the palpated Tuffier's line in parturient and non-parturient women in the lateral decubitus position.

Methods: We consecutively enrolled 40 parturient women at 37-41 weeks of gestation and 40 non-parturient women scheduled for regional anesthesia. In the left lateral position, the location of the vertebra was identified using ultrasonography. We marked every intervertebral space from L5 to L2 vertebra, divided each spinous process into two equal parts, and numbered the spaces sequentially from 1 to 9 . We drew a Tuffier's line by palpating, recorded the vertebral level that this line intersected.

Results: The mean value of an arbitrary number of vertebral level of Tuffier's line was $6.4 \pm 0.9$ in the non-pregnant group and this represents L4-lower vertebral level. In the pregnant group, the mean value was $3.0 \pm 1.0$ which represents L3-lower vertebral level. There was a significant difference between the two groups $(\mathrm{P}<0.05)$.

Conclusions: We compared using an available ultrasound technique the vertebral levels intersected by the palpated Tuffier's line between parturient and non-parturient women and found that the vertebral levels were more cephalad in the parturient women compared to the non-parturient women. (Korean J Anesthesiol 2014; 67: 181-185)

Key Words: Parturient, Spinal anesthesia, Ultrasonography, Vertebra.

Received: April 30, 2014. Accepted: May 27, 2014.

Corresponding author: Dong Yeon Kim, M.D., Department of Anesthesiology and Pain Medicine, Ewha Womans University School of Medicine, 1071, Anyangcheon-ro, Yangcheon-gu, Seoul 158-710, Korea. Tel: 82-2-2650-5558, Fax: 82-2-2655-2924, E-mail: kdyeon@ewha.ac.kr

(c) This is an open-access article distributed under the terms of the Creative Commons Attribution Non-Commercial License (http:// creativecommons.org/licenses/by-nc/3.0/), which permits unrestricted non-commercial use, distribution, and reproduction in any medium, provided the original work is properly cited. 


\section{Introduction}

Development of various anesthetic techniques has allowed for various kinds of surgeries by means of regional anesthesia. In the area of obstetric anesthesia also, spinal and epidural anesthesia are often used [1], which have seen a significant increase in their clinical use. In spinal anesthesia, a needle is generally inserted to the L3-4 intervertebral space or under in order to minimize the danger of spinal cord damage by the needle. Therefore, it is very important to precisely measure the vertebral level.

Tuffier's line is a transverse line connecting the tops of the iliac crests. Generally, it is known that Tuffier's line intersects the spine at the L4 spinous process or at the L4-L5 intervertebral space [2]. Many anesthesiologists generally use this virtual line as an anatomical landmark in spinal and epidural anesthesia. The currently used clinical method is to determine the vertebral level with Tuffier's line based on palpation. However, the vertebral level determined by such a method may be inaccurate. Broadbent et al. [3] predicted the vertebral level of 100 patients and reported that the predicted vertebral level coincided with the actual level verified by magnetic resonance imaging (MRI) only in $29 \%$ of the patients. Duniec et al. [4] reported in a study conducted with 122 patients that the vertebral level verified by ultrasound coincided with the one predicted by anesthesiologists in $64 \%$ of the patients.

Full term parturient women undergo various physical changes including overlordosis and increased weight, and the pelvis of a parturient woman may be rotated to the long axis of the spinal column. Therefore, determining the vertebral level with Tuffier's line based on palpation inevitably is not very accurate.

Recently, the use of ultrasound has increased in regional anesthesia by anesthesiologists. Because ultrasound is noninvasive and free of radiation and can be used in parturient women, it provides much help in accurately determining the vertebral level during spinal and epidural anesthesia in parturient women. In addition, ultrasound could play an important role in preventing complications which could be caused by a wrong prediction of the vertebral level.

The aim of this study was to use ultrasound to verify the difference between vertebral levels for the palpated Tuffier's line in parturient and non-parturient women in the lateral decubitus position. In addition, the correlation of the difference in the measured vertebral levels for various factors such as a patient's physical changes was investigated.

\section{Materials and Methods}

This study was approved by the Institutional Ethics Review Board. The American Society of Anesthesiologists physical status I or II female patients aged $20-45$ years old were selected as sub- jects. We consecutively enrolled 40 parturient women (pregnant group) at 37-41 weeks of gestation who had been scheduled for epidural or combined spinal-epidural anesthesia either for labor or cesarean section. Forty non-parturient women (nonpregnant group) who had been scheduled for surgery with regional anesthesia were also consecutively enrolled. Exclusion criteria included those with a known spinal deformity or had a prior history of spinal surgery, and patients who had difficulty maintaining an appropriate position for the procedure.

After arriving at the operating room, the patients were instructed to lie in the left lateral position with each neck, back, hips and knees flexed maximally. A skilled assistant helped patients to maintain the position. The location of the vertebra was identified using the Sonosite MTurbo (Sonosite ${ }^{\circledR}$, Bothell, WA, USA) ultrasound equipment. The $60 \mathrm{~mm}, 2-5 \mathrm{MHz}$ curved array probe was used. The probe was placed $3 \mathrm{~cm}$ from the midline of the spinal longitudinal plane. And the probe was angled slightly towards the center of the spinal canal, placed on the paramedian longitudinal plane. Beginning at the sacrum, we moved the probe in a cephalad direction slowly. We marked every intervertebral space from L5 to L2 vertebra. Each intervertebral space was considered as one segment. We divided each spinous process into two equal parts. The upper portion was named "upper" and the lower portion was named "lower". Each intervertebral space was recorded as L2-3, L3-4, L4-5. Beginning at the L5 lower vertebra to the L2-3 intervertebral space, we numbered the spaces sequentially from 1 to 9 . The most cephalad part was numbered 1 and the most caudal part was numbered 9, this was an arbitrary division. We drew a line across both iliac crests with a marking pen by palpating the superior aspects of the iliac crests, and then recorded the vertebral level that this line intersected.

The results were presented as the mean \pm SD. Statistical analysis was performed using STATA (version 12.1, STATA Corporation, USA). Student t-test and Spearman's correlation were used. $P$ values less than 0.05 were considered statistically significant.

\section{Results}

The study was conducted with 80 patients, consisting of 40 parturient women and 40 non-parturient women. There were no significant differences in age and height between the two groups. Due to the characteristic of pregnancy, there were significant differences in weight and body mass index (BMI) $(\mathrm{P}<0.05$, Table 1$)$.

The mean value of an arbitrary number of vertebral level of Tuffier's line, measured by ultrasonography, was $6.4 \pm 0.9$ in the non-pregnant group and this represents L4-lower vertebral level. In the pregnant group, the mean value was $3.0 \pm 1.0$ which represents L3-lower vertebral level. There was a significant 
Table 1. Patients' Characteristics

\begin{tabular}{lccc}
\hline & $\begin{array}{c}\text { Nonpregnancy } \\
(\mathrm{n}=40)\end{array}$ & $\begin{array}{c}\text { Pregnancy } \\
(\mathrm{n}=40)\end{array}$ & P value \\
\hline Age $(\mathrm{yr})$ & $35.0 \pm 7.7$ & $33.4 \pm 3.4$ & 0.23 \\
Height $(\mathrm{cm})$ & $161.3 \pm 4.8$ & $162.6 \pm 5.1$ & 0.23 \\
Weight $(\mathrm{kg})$ & $56.4 \pm 7.2$ & $68.2 \pm 6.8$ & $<0.0001$ \\
BMI $\left(\mathrm{kg} / \mathrm{m}^{2}\right)$ & $21.7 \pm 2.8$ & $25.8 \pm 2.4$ & $<0.0001$ \\
Gestational age (week) & & $38.6 \pm 1.3$ & \\
Primiparity & & $24(60 \%)$ & \\
\hline
\end{tabular}

Values are mean \pm SD or number of patients (\%). BMI: body mass index.

Table 2. Correlation Coefficients: between the Vertebral Level of the Tuffier's Line and Patients' Characteristics

\begin{tabular}{lrcrc}
\hline & \multicolumn{2}{c}{$\begin{array}{c}\text { Nonpregnancy } \\
(\mathrm{n}=40)\end{array}$} & \multicolumn{2}{c}{$\begin{array}{c}\text { Pregnancy } \\
(\mathrm{n}=40)\end{array}$} \\
\cline { 2 - 5 } & \multicolumn{1}{c}{$\rho$} & P value & $\rho$ & P value \\
\hline Age $(\mathrm{yr})$ & 0.08 & 0.62 & -0.14 & 0.38 \\
Height $(\mathrm{cm})$ & -0.19 & 0.23 & 0.15 & 0.36 \\
Weight $(\mathrm{kg})$ & -0.43 & 0.01 & -0.06 & 0.72 \\
BMI $\left(\mathrm{kg} / \mathrm{m}^{2}\right)$ & -0.35 & 0.03 & -0.12 & 0.46 \\
\hline
\end{tabular}

$\rho$ : Correlation Coefficients. BMI: body mass index.

difference between the two groups $(\mathrm{P}<0.05)$. This result demonstrates that the vertebral level of Tuffier's line has shifted to cephalad in the pregnant group. In the non-pregnant group, 35 patients (88\%) showed a vertebral level at L4 and L4-5, in the pregnant group, 37 patients (93\%) were at L3 and L3-4, and all patients showed a level lower than L2-3 (Fig. 1).

The vertebral level that intersected at the Tuffier's line was significantly correlated with weight and BMI in the non-pregnant group. However, in the pregnant group, there was no significant correlations were observed (Table 2).

\section{Discussion}

This study showed that vertebral levels measured using ultrasound with the palpated Tuffier's line in parturient women in the lateral decubitus position were more cephalad than those measured in non-parturient women. Anesthesiologists generally determine the needling point for spinal anesthesia with reference to Tuffier's line which is a virtual line connecting the tops of the iliac crests of a patient. Anatomically, it is known that the conus medullaris ends at L1 and L2 vertebra in adults. To minimize damage to the spinal cord, the needling point should be under the conus medullaris in spinal anesthesia. Therefore, it is common for a needle to be inserted under the L3-4 intervertebral space in most spinal anesthesia cases. Saifuddin et al. [5] indicated that previous reports had been obtained from cadaveric studies and reported results based on MRI for which the tip of the conus medullaris was positioned between the

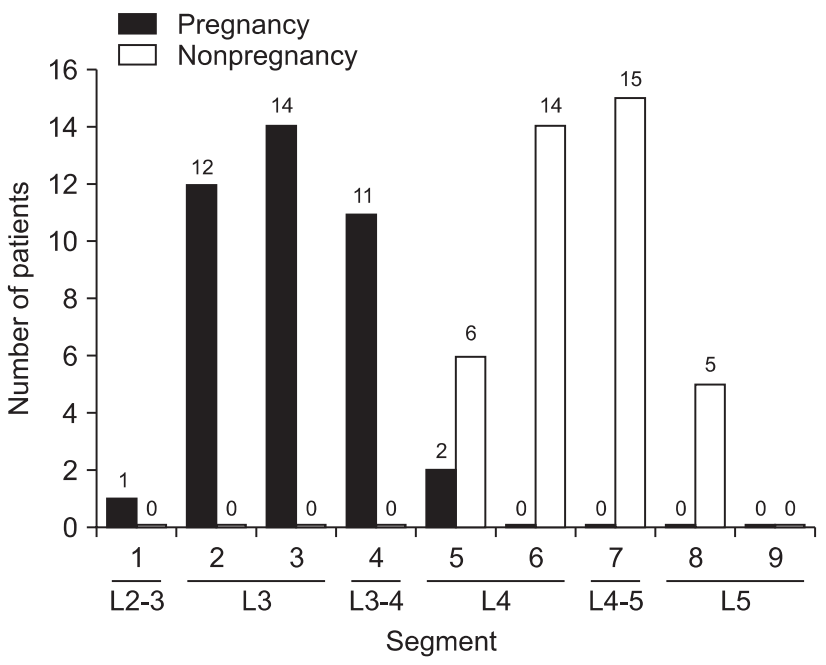

Fig. 1. Distribution of the vertebral level of the intercristal line by ultrasonography.

middle $1 / 3$ point of the T12 vertebral body and the top $1 / 3$ point of the L3 vertebral body in adult patients in the supine position. Kim et al. [6] also reported results based on MRI for which the tip of the conus medullaris was positioned between the top $1 / 3$ point of the T12 vertebral body and the bottom $1 / 3$ point of the L2 vertebral body in female patients and that Tuffier's line was positioned between L3-4 and L5-S1, indicating that the two structures did not overlap in any of the cases. Because the position of Tuffier's line is not accurate in spinal anesthesia, inserting a needle to a position higher than the tip of the conus medullaris could cause spinal damage. Therefore, accurate measurements of the vertebral levels with Tuffier's line are required.

At present, anesthesiologists commonly use the clinical method of performing spinal anesthesia by measuring Tuffier's line using palpation. Regarding the accuracy of Tuffier's line, there is a report that the vertebral levels based on the palpated Tuffier's line were different from those verified by actual radiographic images and that the vertebral levels were higher in patients with a higher BMI [7]. It was also reported that the difficulty of spinal anesthesia was significantly increased when the accuracy of an anatomical index such as Tuffier's line was reduced due to a high BMI [8]. In this study, the vertebral levels based on Tuffier's line were correlated with BMI in the general group of non-parturient women. This result shows that the determined position could be inaccurate in the case of spinal anesthesia performed by measuring the vertebral levels based on the palpated Tuffier's line, demonstrating that the vertebral levels estimated by the Tuffier's line generally should not be applied to all patients.

Pregnancy causes various physical changes. In a full term parturient woman, overlordosis may take place, the pelvis may be rotated to the long axis of the spinal column and body weight may be increased. Several reports have shown that such physi- 
cal changes could make measuring the vertebral levels with Tuffier's line more inaccurate. Margarido et al. [9] measured with ultrasound the vertebral levels with Tuffier's line in full term parturient women while they were in the sitting position and reported that the median of the vertebral levels was the L2-3 intervertebral space which was more cephalad compared to non-parturient women. Locks et al. [10] conducted a study with parturient women who were scheduled to undergo regional anesthesia for cesarean section in which the L3-4 intervertebral space was predicted by palpation while the patients were in the sitting position, and the result was later verified with ultrasound and reported that the vertebral level was accurately predicted only in 53 and $49 \%$ of the patients in the non-obese and obese groups, respectively. Whitty et al. [11] also compared the predicted vertebral level based on the palpated Tuffier's line in parturient women undergoing regional anesthesia while they were in the sitting position with the level measured by ultrasound and reported that the conformity between the two levels was only $55 \%$. In this study also, the vertebral level intersected by Tuffier's line was more cephalad in the parturient women group than that in the non-parturient women group.

Although the general position of patients for spinal anesthesia is the lateral decubitus position, it is well known that performing spinal anesthesia in the sitting position is easier in obese patients because identifying Tuffier's line by palpation and predicting the intervertebral space are much more difficult in obese patients from the inaccuracy of the patients' position and thick subcutaneous tissue. In western countries, spinal anesthesia for full term parturient women is almost always performed in the sitting position because of high body weight. Therefore, most of the reports published until now present results observed when parturient women are in the sitting position. In Asia, especially in Korea, spinal anesthesia for parturient women is performed mostly in the lateral decubitus position because the increase in body weight even in full term parturient women is not severe. Therefore, this study is significant in that spinal anesthesia was performed in parturient women who were in the lateral decubitus position. The finding of this study that the vertebral level intersected Tuffier's line was shifted cephalad could provide great help in performing spinal anesthesia in parturient women. If a needle is inserted only by palpation during spinal or epidural anesthesia in the lateral position, it should be noted that the needle could be inserted into a level higher rather than the estimated vertebral level.

Ultrasound is often used for nerve block because ultrasound is noninvasive and free of radiation [12]. The use of ultrasound in spinal or epidural anesthesia is also increasing [13]. Moreover, ultrasound could be very useful in parturient women in who radiation cannot be applied. However, investigating the structure of the vertebrae with ultrasound is more difficult than investigating the structure of other organs. The vertebrae is a structure deeper than peripheral nerves, and the ultrasound window is very narrow due to the structural characteristics of the vertebrae. It is surrounded by many other structures in contrast to other organs, and individual vertebrae are adjacent to each other. Among various approaches, the paramedian longitudinal approach shows the best acoustic window for spinal ultrasonography, and in the saw-like image obtained through that approach, the saw blades represent articular processes, and the spaces between the saw blades represent the interspaces [14]. In this study also, the paramedian longitudinal approach was used to identify each of the vertebral levels and the interspace.

During spinal anesthesia, the vertebral level through which a needle is inserted is very important because the blocked vertebral level becomes higher as the vertebral level in which the needle is inserted also becomes higher when the same quantity of anesthetic is used [15]. This study showed that Tuffier's line intersects vertebral level more cephalad in parturient women compared to non-parturient women. Therefore, during spinal anesthesia in parturient women, if the vertebral level is determined using the palpated Tuffier's line, a vertebral level higher than the original intended one could be blocked. In addition, more care is required because the possibility of side effects such as spinal damage cannot be excluded, though the possibility is low.

In conclusion, we compared using an available ultrasound technique the vertebral levels intersected by the palpated Tuffier's line between parturient and non-parturient women and found that the vertebral levels were more cephalad in the parturient women compared to the non-parturient women. Therefore, it should be noted during spinal anesthesia in parturient women that a needle could be inserted into a level higher than the estimated vertebral level and more attention should be given to determining the needling point.

\section{References}

1. American Society of Anesthesiologists Task Force on Obstetric Anesthesia. Practice guidelines for obstetric anesthesia: an updated report by the American Society of Anesthesiologists Task Force on Obstetric Anesthesia. Anesthesiology 2007; 106: 843-63.

2. Ellis H, Feldman S, Harrop-Griffiths W. Anatomy for anaesthetists. 8th ed. Malden, Blackwell. 2004, pp 105-6.

3. Broadbent CR, Maxwell WB, Ferrie R, Wilson DJ, Gawne-Cain M, Russell R. Ability of anaesthetists to identify a marked lumbar interspace. Anaesthesia 2000; 55: 1122-6. 
4. Duniec L, Nowakowski P, Kosson D, Łazowski T. Anatomical landmarks based assessment of intravertebral space level for lumbar puncture is misleading in more than 30\%. Anaesthesiol Intensive Ther 2013; 45: 1-6.

5. Saifuddin A, Burnett SJ, White J. The variation of position of the conus medullaris in an adult population. A magnetic resonance imaging study. Spine (Phila Pa 1976) 1998; 13: 1452-6.

6. Kim JT, Bahk JH, Sung J. Influence of age and sex on the position of the conus medullaris and Tuffier's line in adults. Anesthesiology 2003; 99: 1359-63.

7. Chakraverty R, Pynsent P, Isaacs K. Which spinal levels are identified by palpation of the iliac crests and the posterior superior iliac spines? J Anat 2007; 210: 232-6.

8. Kim JH, Song SY, Kim BJ. Predicting the difficulty in performing a neuraxial blockade. Korean J Anesthesiol 2011; 61: 377-81.

9. Margarido CB, Mikhael R, Arzola C, Balki M, Carvalho JC. The intercristal line determined by palpation is not a reliable anatomical landmark for neuraxial anesthesia. Can J Anaesth 2011; 58: 262-6.

10. Locks Gde F, Almeida MC, Pereira AA. Use of the ultrasound to determine the level of lumbar puncture in pregnant women. Rev Bras Anestesiol 2010; 60: 13-9.

11. Whitty R, Moore M, Macarthur A. Identification of the lumbar interspinous spaces: palpation versus ultrasound. Anesth Analg 2008; 106 : 538-40.

12. Jung H, Kim DH, Jeon SH, Kim CY, Kim JS, Choi YS. The Effectiveness of ultrasound guidance in caudal epidural block. J Korean Soc Spine Surg 2013; 20: 178-83.

13. Pak MH, Lee WH, Ko YK, So SY, Kim HJ. Ultrasonographic measurement of the ligamentum flavum depth; is it a reliable method to distinguish true and false loss of resistance? Korean J Pain 2012; 25: 99-104.

14. Carvalho JC. Ultrasound-facilitated epidurals and spinals in obstetrics. Anesthesiol Clin 2008; 26: 145-58.

15. Tuominen M, Taivainen T, Rosenberg PH. Spread of spinal anaesthesia with plain $0.5 \%$ bupivacaine: influence of the vertebral interspace used for injection. Br J Anaesth 1989; 62: 358-61. 